\title{
Prácticas de examen en línea en una institución de educación superior del Perú
}

\section{Online exam practices in a higher education institution in Peru}

\author{
Roberto Carlos Dávila Morán \\ rdavila430@gmail.com \\ https://orcid.org/0000-0003-3181-8801 \\ Universidad Privada del Norte, Lima, Perú. \\ Eucaris del Carmen Agüero Corzo \\ caricorzo@gmail.com \\ https://orcid.org/0000-0003-4587-3852 \\ Universidad Pedagógica Experimental Libertador, Maturín, Venezuela.
}

Recibido: $10 / 08 / 21$

Aceptado: 25/10/21

\section{Resumen}

El propósito de este estudio fue examinar las percepciones de los estudiantes sobre las prácticas de exámenes en línea en una Universidad del Perú. La investigación es de diseño no experimental, de tipo básica; se empleó como instrumento el cuestionario en línea aplicado a 432 estudiantes de pregrado. Los resultados muestran la percepción que los exámenes en línea tienen beneficios significativos sobre los exámenes tradicionales basados en papel, la confiabilidad de la calificación y la eficiencia en términos de tiempo, esfuerzo y dinero. La implementación exitosa de éstos depende de diseñarlos para que sean válidos, confiables, seguros y flexibles.

Palabras clave: Examen en línea, educación superior, percepción de los estudiantes.

\section{Abstract}

The purpose of this study was to examine students' perceptions about online exam practices at a University in Peru. The research is of a non-experimental design, of a basic type; The online questionnaire applied to 432 undergraduate students was used as an instrument. The results show the perception that online exams have significant benefits over traditional paper-based exams, scoring reliability, and efficiency in terms of time, effort, and money. Their successful implementation depends on designing them to be valid, reliable, secure, and flexible.

Keywords: Online exam, higher education, student perception. 


\section{Introducción}

Como resultado de la avanzada difusión de las tecnologías de la información con la creciente disponibilidad de opciones de plataforma de evaluación virtual, los exámenes en línea se están implementando cada vez más en las instituciones de educación superior (IES) como un medio para complementar o incluso reemplazar las pruebas en papel (Nicol, 2007). Los mismos, son comúnmente conocidos como exámenes electrónicos y anteriormente como evaluación basada en computadoras, pueden definirse como "un sistema que implique la realización de evaluaciones a través de la web o la intranet" (Ayo et al., 2007, p. 126). Funcionalmente, se pueden entregar utilizando un sistema dedicado o incluirse como un learning management system (LMS) como Blackboard, Moodle o Sakai (Sorensen, 2013). El módulo de actividad del examen permite al profesor diseñar y establecer exámenes que consisten en una variedad de tipos de preguntas, incluyendo opciones múltiples, verdadero-falso y de respuesta corta. Estos se mantienen en un banco de preguntas y se puede reutilizar en varios exámenes. Los exámenes se pueden configurar para permitir múltiples intentos. Por lo tanto, los sistemas de exámenes en línea permiten simplificar el proceso tradicional de exámenes basados en papel, especialmente cuando los tamaños de clase son grandes, desde el diseño y la aplicación del examen para marcar, informar, almacenar los resultados y efectuar análisis estadísticos (Osuji, 2012; Farzin, 2017). Hay un conjunto de investigaciones que destacan una serie de beneficios clave de los exámenes en línea, así como algunos desafíos notables desde las perspectivas de profesores y estudiantes (Nicol, 2007; Dermo, 2009; Dreher et al., 2011; Hodgson y Pang, 2012; James, 2016).

Entre sus principales ventajas sobre los exámenes en papel se encuentran que constituyen un sistema totalmente automatizado el cual mejora la validez de la evaluación, donde el rendimiento del examinado es una medida justa de habilidad y comprensión y que utiliza estilos de preguntas mejorados que incorporan interactividad y multimedia. Los exámenes en línea mejoran la fiabilidad de la puntuación y la robustez de los resultados de las pruebas, apoyando un análisis más profundo. Ellos también proporcionan retroalimentación inmediata, corrigiendo así rápidamente los conceptos erróneos. En comparación con los exámenes basados en papel, el uso de exámenes en línea puede mejorar significativamente la eficiencia de las tareas de gestión de datos, como marcar, moderar y almacenar información, reduciendo así la carga de trabajo de los docentes, que ahorran tiempo dedicado a la rutina de trabajo, al mismo tiempo que reduce significativamente la carga asociada con la vigilancia cuando se evalúan grandes cohortes.

\section{Metodología}

El estudio se desarrolló con un diseño no experimental de tipo básica (Hernández Sampieri et al., 2010). La técnica para el recojo de datos fue la encuesta; el Cuestionario de Percepción de La Evaluación Electrónica del Estudiante (Dermo, 2009) se utilizó como base sobre la cual elaborar un cuestionario en línea que consta de tres partes principales. En la primera parte se preguntó a los participantes por información. La segunda parte les 
pidió que respondieran a una serie de declaraciones relativas a sus opiniones sobre los exámenes en línea, todas redactadas positivamente y agrupadas en seis conjuntos: pedagogía, validez, confiabilidad, factores afectivos, practicidad y seguridad. La tercera parte pidió a los participantes que seleccionaran las consideraciones importantes para la implementación exitosa de exámenes en línea. También se les pidió que comentaran sobre los temas abordados por el cuestionario en una cuarta sección. La validez del contenido del cuestionario se evaluó pidiendo a tres expertos en e-learning que emitan sus juicios sobre los elementos. Se recibieron comentarios positivos y se hicieron algunas revisiones menores al instrumento de acuerdo con sus sugerencias. La fiabilidad de los constructos (en la medida en que los ítems del cuestionario estaban relacionados entre sí) se examinó utilizando el alfa de Cronbach. Todos los valores estuvieron por encima de 0.70 , lo cual es bueno. La encuesta se realizó del 17 de mayo de 2021 al 31 de mayo de 2021. Los participantes eran estudiantes de pregrado en cuatro disciplinas: Ingeniería, Administración, Derecho y Economía.

\section{Perfil de los participantes}

Hubo 432 estudiantes encuestados: $56.02 \%$ mujeres y $43.98 \%$ hombres. El grupo más grande fue de la disciplina de Ingeniería (39.1\% de los encuestados), seguido de Administración (31.4\%), Derecho (16.1\%) y Economía (13.4\%). El 70\% se describió a sí mismo como no tener experiencia previa de exámenes en línea y el 30\% había tomado sólo un examen en línea. Esta variación parece reflejar la realidad sobre el terreno; la mayoría de los que tomen las pruebas eran estudiantes de primer año registrados en cursos básicos.

\section{Resultados}

Los encuestados reportaron experiencias mixtas de exámenes en línea. De los 18 puntos del cuestionario, siete recibieron respuestas medias positivas, ocho tenían respuestas claramente negativas y tres tenían respuestas neutrales en general (ver tabla 1). Estas clasificaciones se basan en una escala de Likert adaptada de cinco puntos, donde el valor medio de 3 puede ser visto como una posición neutral, mientras que un valor medio por encima de esto se puede ver como positivo y una calificación media por debajo de 3 se considera negativo (Dermo, 2009).

Para los estudiantes, el aspecto más ventajoso de los exámenes en línea era que la calificación se consideraba más precisa que los métodos tradicionales (media $=4.3$ ). $A$ continuación, se consideraron más eficientes en términos de tiempo, esfuerzo y dinero gastado en el proceso de examen (media $=4.1$ ). Las preocupaciones más fuertes para los estudiantes, reflejadas respuestas negativas, fueron la imparcialidad de los bancos de preguntas (media $=1.7$ ) y si la tecnología era lo suficientemente eficaz para hacer frente a las trampas y el plagio (media $=1.6$ ). 
Revista de Tecnología de Información y Comunicación en Educación • Volumen 15, ํㅜ 3. Septiembre-diciembre 2021

Tabla 1.

Respuestas de los participantes a las ventajas de los exámenes en línea.

\begin{tabular}{|c|c|c|c|c|c|c|}
\hline Elementos del cuestionario & MA & DA & NS & ED & TA & Media \\
\hline 1. Pedagogía & $\%$ & $\%$ & $\%$ & $\%$ & $\%$ & \\
\hline $\begin{array}{l}\text { La retroalimentación inmediata en los } \\
\text { exámenes en línea ayuda a los } \\
\text { estudiantes a profundizar } \\
\text { comprensión de la asignatura }\end{array}$ & 34.7 & 18.3 & 9.3 & 21.8 & 15.9 & 3.2 \\
\hline $\begin{array}{l}\text { El uso de la tecnología más avanzada } \\
\text { en los exámenes en línea permite a los } \\
\text { estudiantes adoptar un nuevo enfoque } \\
\text { de aprendizaje: el aprendizaje en línea }\end{array}$ & 17.4 & 63.2 & 7.2 & 9.7 & 2.5 & 3.9 \\
\hline
\end{tabular}

Los exámenes en línea facilitan un enfoque de aprendizaje más adaptativo

$\begin{array}{llllll}8.6 & 27.8 & 9 & 38.4 & 16.2 & 2.8\end{array}$ que los de papel

2. Validez

$\begin{array}{lllllll}\text { Los exámenes en línea son apropiados } & 5.3 & 8.3 & 11.3 & 27.3 & 47.7 & 1.9\end{array}$ para cualquier materia

$\begin{array}{lllllll}\text { Los exámenes en línea son adecuados } & 2.3 & 4.6 & 12.3 & 29.6 & 51.2 & 1.8\end{array}$ para comprobar el nivel de conocimientos de los estudiantes

$\begin{array}{llllllll}\text { Los exámenes en línea facilitan una } & 28.5 & 41.4 & 12.3 & 12.5 & 5.3 & 3.7\end{array}$ evaluación más auténtica que los métodos tradicionales mediante la integración de multimedia, simulaciones

3. Fiabilidad

La corrección automática de los exámenes en línea es más precisa que la corrección en papel

$\begin{array}{lllllll}\text { Los exámenes en línea son más justos } & 1.2 & 3.2 & 17.1 & 21.1 & 57.4 & 1.7\end{array}$ que los de papel

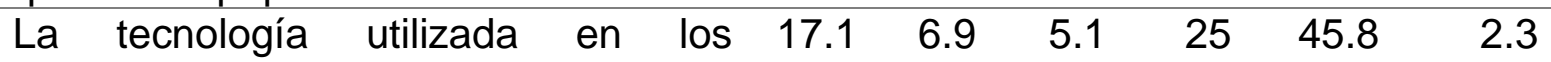
exámenes en línea es fiable

4. Factores afectivos

$\begin{array}{llllllll}\text { Los exámenes en línea reducen el } & 14.1 & 9.3 & 3.0 & 46.5 & 27.1 & 2.4\end{array}$ estrés y la ansiedad de los exámenes

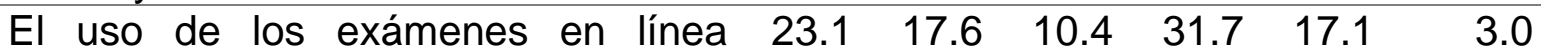
permite a los estudiantes centrarse y concentrarse más en las preguntas 


\begin{tabular}{|c|c|c|c|c|c|c|}
\hline \multirow{2}{*}{\multicolumn{7}{|c|}{$\begin{array}{l}\text { Los estudiantes se sienten más } \\
\text { cómodos haciendo un examen en línea } \\
\text { que en papel } \\
\text { 5. Practicidad }\end{array}$}} \\
\hline & & & & & & \\
\hline $\begin{array}{l}\text { eficientes en términos de tiempo, } \\
\text { esfuerzo y dinero gastado }\end{array}$ & 42.0 & 34.1 & 0.9 & 1.9 & 1.9 & 4.1 \\
\hline $\begin{array}{l}\text { La creación de un banco de preguntas } \\
\text { de opción múltiple reutilizables permite } \\
\text { un fácil almacenamiento y revisión }\end{array}$ & 16.2 & 32.2 & 7.2 & 20.1 & 24.3 & 2.9 \\
\hline $\begin{array}{l}\text { Los exámenes en línea son más } \\
\text { accesibles que los de papel }\end{array}$ & 25.7 & 45.4 & 9.7 & 8.6 & 10.6 & 3.6 \\
\hline 6. Seguridad & & & & & & \\
\hline $\begin{array}{l}\text { Los materiales de prueba y los } \\
\text { resultados de los exámenes en línea } \\
\text { son más seguros que los métodos } \\
\text { tradicionales }\end{array}$ & 15.5 & 28.5 & 11.1 & 17.6 & 27.3 & 2.9 \\
\hline $\begin{array}{l}\text { La tecnología utilizada en los } \\
\text { exámenes en línea es lo } \\
\text { suficientemente eficaz para tratar las } \\
\text { trampas y el plagio }\end{array}$ & 3.7 & 3.0 & 10.2 & 18.1 & 65 & 1.6 \\
\hline $\begin{array}{l}\text { El uso de preguntas aleatorias de un } \\
\text { banco significa que las trampas } \\
\text { durante los exámenes en línea es } \\
\text { menos probable que en los de papel }\end{array}$ & 23.1 & 52.3 & 4.2 & 8.1 & 12.3 & 3.6 \\
\hline
\end{tabular}

La encuesta también enumeró varias consideraciones que podrían mejorar la implementación de exámenes en línea. Se pidió a los participantes que seleccionaran elementos de una lista de verificación, como se muestra en la tabla 2.

Tabla 2.

Consideraciones para la aplicación efectiva de los exámenes en línea.

\begin{tabular}{|c|c|c|}
\hline Consideraciones & Frecuencia & $\%$ \\
\hline 1. Diseño de examen en línea & & \\
\hline $\begin{array}{l}\text { Mantener un banco de preguntas validadas para las } \\
\text { pruebas adaptativas }\end{array}$ & 370 & 85.6 \\
\hline Desarrollar diferentes tipos de preguntas tipo & 365 & 84.5 \\
\hline $\begin{array}{l}\text { Proporcionar una retroalimentación significativa e } \\
\text { inmediata }\end{array}$ & 378 & 87.5 \\
\hline 2. Seguridad en los exámenes en línea & & \\
\hline Mantener la confidencialidad & 367 & 85.0 \\
\hline Minimizar las trampas & 270 & 62.5 \\
\hline Autenticación & 312 & 72.2 \\
\hline
\end{tabular}


Geduweb

Revista de Tecnología de Información y Comunicación en Educación • Volumen 15, № 3. Septiembre-diciembre 2021

3. Propósito del examen en línea

\begin{tabular}{lll} 
Examen formativo para el aprendizaje & 310 & 71.8 \\
\hline $\begin{array}{l}\text { Vinculación de los exámenes en línea con los resultados de } \\
\text { aprendizaje previstos }\end{array}$ & 325 & 75.2 \\
$\begin{array}{l}\text { Vincular el análisis de los resultados a los criterios de } \\
\text { garantía de calidad }\end{array}$ & 298 & 69.0 \\
\hline
\end{tabular}

4. Apoyo institucional

Integración del examen en línea en el plan estratégico

321

74.3

Proporcionar recursos y facilitar procedimientos

303

70.1

Apoyo a profesores y estudiantes

337

78.0

\section{Discusión}

En general, los participantes parecían considerar las prácticas de exámenes en línea actualmente implementadas por la Universidad Alas Peruanas insatisfactorio. Aunque identificaron varias características positivas de los exámenes en línea, incluyendo la fiabilidad de calificación y eficiencia en términos de tiempo, esfuerzo y costo, también señalaron muchos desafíos a los que se enfrenta la implementación exitosa de exámenes en línea en áreas tales como seguridad, validez y equidad. En consecuencia, la encuesta ha identificado una serie de posibles mejoras en los exámenes en línea en la Universidad Alas Peruanas y, en general, entre las instituciones de educación superior (IES) peruanas, desde la perspectiva de los estudiantes.

\section{Pedagogía}

Desde una perspectiva teórica, se ha asumido que la retroalimentación inmediata es una ventaja fundamental pedagógicamente de los exámenes en línea, según lo informado por varios estudios (Heinrich et al., 2009; Dreher et al., 2011; Hodgson y Pang, 2012; Kuikka et al., 2014). La tabla 1 muestra que sólo el $53 \%$ de los encuestados a este estudio estuvieron de acuerdo o totalmente de acuerdo en firmemente en que la retroalimentación inmediata en los exámenes en línea ayuda a los estudiantes a obtener una comprensión más profunda del tema. La falta de reconocimiento de la utilidad de la retroalimentación puede ser el resultado del hecho de que el examen en línea actualmente implementado en la Universidad Alas Peruanas es exclusivamente sumativo y no proporciona una evaluación formativa para que los estudiantes no reciban comentarios. Como la retroalimentación inmediata puede ayudar a corregir conceptos erróneos y mejorar el aprendizaje de los estudiantes, es indispensable dar a los estudiantes retroalimentación inmediata y significativa sobre las evaluaciones sumativas y formativas (Hodgson y Pang, 2012; Kuikka et al., 2014). La tabla 2 muestra que el $87.5 \%$ de los participantes reconoció la importancia de la retroalimentación como una consideración crucial que podría mejorar la implementación del examen en línea, siendo esto la respuesta más fuerte a un ítem de la tercera sección del cuestionario. Más de dos tercios $(71.8 \%)$ de los participantes también consideraron que los exámenes en línea son adecuados para la evaluación formativa para medir el aprendizaje, en lugar de una 
evaluación del aprendizaje. Por lo tanto, uno de los objetivos del examen en línea debe ser promover el aprendizaje mediante la provisión de comentarios constructivos y en tiempo real. Un examen en línea no es sólo una herramienta de calificación automatizada; también es parte integral del proceso de aprendizaje en sí (Jordan, 2013). Por lo tanto, la retroalimentación efectiva debe centrarse en ayudar a los estudiantes a adquirir una comprensión clara de los conceptos y reforzar sus conocimientos. El desafío de proporcionar retroalimentación relevante, oportuna y profunda a los estudiantes radica en el considerable trabajo que se necesitará para capacitar al personal académico y apoyarlos en el desarrollo de una retroalimentación de calidad, adoptando un enfoque más creativo y eficiente, como el uso de audio (McGarvey y Haxton, 2011) y comentarios en video en conjunto con comentarios escritos.

Tres cuartas partes de todos los participantes estuvieron de acuerdo o muy de acuerdo en que el uso de tecnología de vanguardia en los exámenes en línea permite a los estudiantes tomar un nuevo enfoque de aprendizaje: e-learning. Esto es consistente con los hallazgos de otras investigaciones (Dermo, 2009). Es probable que los estudiantes expertos en tecnología prefieran el aprendizaje digital que satisfaga sus necesidades. Como los exámenes en línea son parte de un enfoque de aprendizaje que se ha convertido en la corriente principal de las universidades, el personal académico debe alejarse de sus métodos tradicionales en el aula y adoptar nuevos métodos de integración de las tecnologías emergentes en la enseñanza y aprendizaje, sin embargo, la Universidad Alas Peruanas ha sido lento para adoptar plenamente el aprendizaje en línea. Se necesita tiempo, esfuerzo y dinero para desarrollar la infraestructura, las habilidades, las actitudes y la política para facilitar este enfoque. Es necesario el apoyo institucional para avanzar en el desarrollo sostenible del aprendizaje en línea. Aproximadamente dos tercios de los que respondieron al cuestionario confirmaron la importancia de integrar los procesos de evaluación y aprendizaje en línea dentro de los planes estratégicos de la universidad.

Una característica importante de los exámenes en línea es la adaptabilidad de las pruebas para satisfacer las diversas necesidades de los estudiantes. Las pruebas adaptativas, en las que el programa informático adapta automáticamente la siguiente pregunta en función del rendimiento de cada estudiante en las secciones anteriores de la evaluación, se consideraron problemáticas, sin embargo, solo el $36.4 \%$ de los participantes opinó que el software de exámenes en línea facilitó un mayor avance en las pruebas adaptativas para los estudiantes y respondía a sus diversas necesidades de aprendizaje. La razón de la percepción general negativa (media $=2.8$ ) puede deberse a que los participantes no habían entendido del todo el concepto de prueba adaptativa, ya que las pruebas que conocían en la Universidad Alas Peruanas eran de formato tradicional. Moodle y otros paquetes de software ofrecen varias funcionalidades de exámenes en línea, pero parece que el comité de exámenes y el personal no eran conscientes de su valor pedagógico. Esto ilustra la necesidad de actualizar la versión de Moodle de forma consistente e instalar todos los plugins necesarios, como la retroalimentación de las tareas y el cuestionario adaptativo, que permite a los profesores crear pruebas que midan eficazmente las capacidades de cada participante. Además, 24 Prácticas de examen en línea en una institución de educación superior del Perú. - Eduweb, 2021,
septiembre-diciembre, v.15, n.3./18-32 
Geduweb

Revista de Tecnología de Información y Comunicación en Educación • Volumen 15, Nㅜ 3. Septiembre-diciembre 2021

para ampliar sus funcionalidades, otros programas de exámenes deberían integrarse en Moodle (o en cualquier LMS). Por último, para hacer los exámenes en línea más eficientes, el personal debería estar capacitado para desarrollar preguntas de prueba adaptativas.

\section{Validez}

Uno de los componentes más importantes de la evaluación es la validez del examen. La tabla 1 muestra que los participantes perciben que los exámenes en línea no son válidos, ya que no son apropiados para muchas asignaturas (75\%) y no están bien para evaluar las habilidades de pensamiento de orden superior (80.8\%). Una crítica común a las preguntas de opción múltiple es que evalúan el conocimiento de los hechos, pero no la comprensión. Un estudiante que no esté seguro de la respuesta correcta puede simplemente adivinar, como observó un participante. Esta conclusión es similar a la de otros investigadores (Hodgson y Pang, 2012). Refleja la necesidad de encontrar formas innovadoras de desarrollar diferentes tipos de preguntas que midan con precisión y apoyen el nivel de conocimientos de los estudiantes. Un examen en línea debe ir más allá de los ítems de opción múltiple, que deben utilizarse en combinación con otros tipos. Cuatro quintas partes de los participantes consideran que esto es una consideración crítica para la implantación efectiva de los exámenes en línea. Los exámenes en línea, especialmente para los cursos de idiomas, deben incluir elementos en los que los estudiantes escuchen material de audio y luego respondan a preguntas sobre el mismo, y otros en los que tengan que dar respuestas orales grabadas en vídeo. Otros tipos de preguntas que también deben incluirse son ensayos cortos y trabajos escritos que demuestren la comprensión de los estudiantes del material y el pensamiento crítico. Una de las principales dificultades en el diseño de los exámenes en línea es crear preguntas no objetivas para medir la comprensión profunda de los estudiantes. Se requiere un trabajo considerable para preparar al personal, no sólo técnicamente sino también pedagógicamente, para diseñar mejor las preguntas de opción múltiple y otros tipos de preguntas que realmente evalúen los resultados de aprendizaje previstos del curso, incluyendo el aprendizaje de orden superior (Kuikka et al., 2014).

La tabla 1 muestra que dos tercios de los participantes (69.6\%) están de acuerdo o muy de acuerdo en que los exámenes en línea facilitan una evaluación más auténtica que los métodos tradicionales gracias a la integración de multimedia y simulaciones. Chua y Don (2013) y Kuikka et al. (2014) informan de resultados similares y concluyen que las nuevas tecnologías permiten exponer a los examinados a vídeos, audios o simulaciones antes de responder a diferentes tipos de preguntas relacionadas con el material multimedia, lo que hace que los exámenes en línea sean más atractivos que los métodos tradicionales. Los estudiantes valoran el aprovechamiento de la tecnología para transformar las prácticas de evaluación y garantizar que ésta sea válida y auténtica, mediante estrategias como la simulación, los enfoques basados en problemas, portafolios de pruebas y la integración de la evaluación en línea y presencial. La tabla 2 muestra que dos tercios de los encuestados reconocen la importancia de la calidad en los exámenes en línea al estar 
de acuerdo en que el análisis de los resultados debe estar vinculado a criterios de garantía de calidad.

\section{Fiabilidad}

La fiabilidad es una cuestión importante en cualquier examen. Cuando se preguntó a los participantes si pensaban que los exámenes en línea mejoraba la exactitud de los resultados, más de tres cuartas partes estuvieron de acuerdo en que la corrección automática es más precisa que la corrección en papel. Un participante comentó que los exámenes en línea son justos y no tienen ningún sesgo en la calificación. Esto coincide con las conclusiones de Baleni (2015), que también encontró que la corrección transparente y la entrega inmediata de las calificaciones dan a los estudiantes más confianza en los resultados que en los de los exámenes tradicionales. No obstante, muchos participantes se mostraron preocupados por la exactitud de los resultados debido a errores en las preguntas y respuestas. Uno de ellos comento que las preguntas incorrectas no se pueden cambiar durante el examen y nos vemos obligados a dar respuestas erróneas. Cualquier ambigüedad en las preguntas invalidará la prueba. Por lo tanto, cada pregunta debe ser revisada por expertos en la materia y el personal académico debe asumir la plena responsabilidad del proceso de evaluación. Esto se refleja en el primer item de la tabla 2 , donde cuatro quintas partes de los encuestados consideran que el éxito de la implantación de los exámenes en línea requiere el mantenimiento de bancos de preguntas validadas. Aunque la mayoría considera que los resultados de los exámenes en línea son más precisos, una proporción aún mayor (78.5\%) de los participantes no está de acuerdo con que los exámenes en línea sean más justos que los basados en papel. Creían que la aleatorización de las preguntas significaría que a algunos estudiantes se les podrían hacer preguntas relativamente fáciles y a otros más difíciles. Esta conclusión coincide con otros estudios (Dermo, 2009; Farzin, 2017). Así pues, para garantizar que las preguntas de los exámenes en línea evalúen adecuadamente los mismos resultados de aprendizaje previstos para todos los estudiantes y tengan una dificultad equivalente (Jordan, 2013). Es necesario considerar las pruebas adaptativas, donde se seleccionan diferentes tipos de preguntas de un banco de preguntas y se utilizan herramientas algorítmicas para asignarlas a niveles de dificultad (Gershon, 2005).

La infraestructura informática existente en la Universidad Alas Peruanas puede no ser lo suficientemente robusta como para hacer frente al creciente número de estudiantes que realizan exámenes en línea. Los exámenes están sujetos de interrupción debido a la lentitud de los ordenadores, a la lentitud de la carga, a la mala conectividad de la red o a las interrupciones del suministro eléctrico de energía. Cuando se produce uno de estos problemas técnicos, hay que reprogramar los exámenes, lo que resulta inconveniente y estresante para los estudiantes. Las universidades deberían mejorar su infraestructura y proporcionar laboratorios totalmente equipados con recursos de seguridad adecuados (Osuji, 2012). Casi dos tercios de los participantes indicaron que el éxito de la implantación de los exámenes en línea depende del apoyo institucional, que incluye la creación de condiciones adecuadas para la realización de los exámenes, la facilitación

\footnotetext{
26 Prácticas de examen en línea en una institución de educación superior del Perú. - Eduweb, 2021,
} 
๔eduweb

Revista de Tecnología de Información y Comunicación en Educación • Volumen 15, Nº 3. Septiembre-diciembre 2021

de los procedimientos administrativos y la provisión de los recursos financieros necesarios para la mejora de las infraestructuras.

\section{Factores afectivos}

Otro ámbito importante de preocupación fueron los aspectos afectivos de los exámenes en línea. Tres cuartas partes de los participantes no estaban de acuerdo con la afirmación de que los exámenes en línea reducen el estrés y la ansiedad. Este resultado está en línea con el trabajo de Bernik y Jereb (2006) y Whitelock (2006). Los participantes mencionaron en sus comentarios varias razones de su estrés, como la interrupción de los exámenes por problemas técnicos, su desconocimiento con el uso de Moodle para los exámenes en línea, su falta de conocimientos informáticos y la mala redacción de las preguntas. Un participante se quejó de que los estudiantes no recibían suficientes instrucciones al principio de los exámenes en línea, un problema agravado por el hecho de que Moodle está en inglés. Como la mayoría de los participantes (70\%) era la primera vez que realizaban un examen en línea, no estaban familiarizados con la plataforma de exámenes en línea y una clara comprensión de sus prácticas (James, 2016). Por lo tanto, es importante que las instituciones presten más atención a la formación tanto del personal académico como de los estudiantes en el proceso de examen en línea antes de que realmente participen en eso (Osuji, 2012; Jordan, 2013). La tabla 2 muestra que el $78 \%$ de los participantes considera que el apoyo a los profesores y a los estudiantes es importante para la aplicación eficaz de los exámenes en línea. Los pocos minutos de orientación dado a los estudiantes antes del examen fue insuficiente para proporcionar instrucciones suficientemente claras. El personal académico necesita tiempo y conocimientos especializados para preparar preguntas de calidad, proporcionar comentarios y gestionar los exámenes en formatos digitales.

Una clara mayoría (61.2\%) de los participantes está de acuerdo con las conclusiones de Bernik y Jereb (2006), en que los estudiantes se sienten más cómodos haciendo un examen en línea que uno en papel, quizás porque este formato reduce considerablemente la necesidad de vigilar el examen. Por el contrario, las respuestas fueron neutrales en general a la sugerencia de que el formato en línea permite a los estudiantes centrarse mejor en las preguntas. Una razón que dio un participante para estar en desacuerdo con esto fue que podía ser difícil concentrarse en la pantalla debido a la necesidad de utilizar papel para resolver problemas específicos, especialmente los que requieren un cálculo o manipulación algebraica. También señaló que, dado que la puntuación se otorga únicamente solo por elegir la opción correcta, un estudiante que eligiera la incorrecta no podría ser recompensado por seguir el método correcto.

\section{Practicidad}

Los resultados también muestran que el $77.3 \%$ de los encuestados está de acuerdo en que los exámenes en línea son más eficientes que los de papel en términos de tiempo, esfuerzo y coste. El proceso totalmente automatizado puede eliminar o simplificar la 
impresión, la calificación, el análisis de los resultados, la vigilancia y la carga de trabajo del personal, especialmente en el caso de clases de gran tamaño. Este hallazgo está alineado con los de varios estudios (Dermo, 2009; Dreher et al., 2011; Baleni, 2015). Sin embargo, para hacer uso de la tecnología en línea para reducir la carga de trabajo del personal, primero es necesario migrar de un papel a un enfoque digital de la pedagogía y el aprendizaje, que es un proceso largo y costoso, especialmente en la fase inicial de la implantación (Jamil et al., 2012; Kuikka et al., 2014).

Podría decirse que otra de las ventajas de los exámenes en línea es el ahorro de tiempo que supone la creación de bancos de preguntas de control reutilizables, de preguntas de opcion multiple reutilizables, que se pueden almacenar y revisar fácilmente para su uso en cohortes posteriores, aunque los encuestados se dividieron casi por igual entre los que estaban de acuerdo y los que no lo estaban (media $=2.9$ ). Los elementos de la prueba deben renovarse para reducir el riesgo de trampas y de memorización. Las preguntas de los exámenes en línea también deben estar sujetas a normas de garantía de calidad, garantizando que el diseño de las preguntas se ajuste a los objetivos de aprendizaje del curso.

Aunque los únicos exámenes en línea que se realizan actualmente en la Universidad Alas Peruanas son exámenes sumativos que se llevan a cabo en los laboratorios de computación del campus, el $71.1 \%$ de los participantes percibieron que los exámenes en línea eran más accesibles que los basados en papel. Las pruebas formativas deben realizarse de forma regular. Los exámenes en línea pueden realizarse en cualquier momento y lugar, incluso durante las clases, y pueden combinarse con trozos de aprendizaje relevantes aprovechando los dispositivos móviles de los estudiantes (Shraim y Crompton, 2015). Un factor relevante es el reciente y significativo desarrollo y expansión de la educación en línea y a distancia. El panorama educativo de Perú está experimentando una transformación en la que las universidades se encuentran en diferentes fases de adopción del aprendizaje y los exámenes en línea. Para que más universidades adopten este enfoque, hay que tener muy en cuenta la flexibilidad en la realización de los exámenes en línea, la mejora de la infraestructura y abordar los problemas de seguridad.

\section{Seguridad}

La seguridad es un aspecto crítico de cualquier examen. La tabla 1 muestra que los participantes están divididos más o menos por igual en cuanto a si los materiales y los resultados de los exámenes son más seguros cuando los exámenes se realizan en línea en lugar de los métodos tradicionales. Esta conclusión es coherente con las de Bernik y Jereb (2006) y Dermo (2009). Los sistemas de gestión de exámenes, como Moodle, incorporan funciones de supervisión para proteger los datos de accesos no autorizados mediante el seguimiento y el registro sistemáticos de actividades como el inicio y el cierre de sesión, el acceso al examen, la navegación por las preguntas y las respuestas. El contenido de cada examen se guarda de forma segura en una base de datos en un servidor accesible sólo al personal autorizado. Como se observa en la tabla 2, el 85\% de

\footnotetext{
28 Prácticas de examen en línea en una institución de educación superior del Perú. - Eduweb, 2021,
} 
los participantes considera muy importante la confidencialidad y la seguridad de la red de los exámenes en línea para evitar el uso indebido de los bancos de preguntas y otros datos, que deben almacenarse en un formato cifrado de alta seguridad.

Además, la respuesta más fuertemente negativa (media $=1.6$ ) fue a la afirmación de que la tecnología de los exámenes en línea es suficientemente eficaz para hacer frente a las trampas y al plagio, con la que hasta el $83.1 \%$ de los participantes estaba en desacuerdo y sólo el $6.7 \%$ estaba de acuerdo. Evitar las trampas en los exámenes en línea puede ser difícil, dada la disponibilidad de tecnologías como Bluetooth, redes inalámbricas, teléfonos móviles y dispositivos portátiles. Estos proporcionan varias formas de buscar en Internet y de comunicarse con otros durante los exámenes y no son fáciles de bloquear. Además, con grupos grandes de estudiantes que se examinan en diferentes momentos, un grupo puede beneficiarse de otro al transmitirse información sobre el examen. Por lo tanto, hacer trampa puede ser difícil de prevenir, sobre todo porque los estudiantes expertos en tecnología siempre encontrarán formas innovadoras de hacer trampa. Aunque la Universidad Alas Peruanas ha promulgado recientemente una normativa contra el uso del teléfono móvil, exigiendo a los estudiantes que lo apaguen durante exámenes, varios participantes mencionaron comportamientos poco éticos en sus comentarios. Un estudiante menciono que se le hace más fácil hacer trampa en un examen en línea porque a menudo uso mi reloj inteligente para enviar una captura de pantalla a todo el grupo. Otro estudiante comentó que utiliza su teléfono móvil para enviar mensajes de texto y chatear con mis amigos. Por lo tanto, es un reto encontrar formas de minimizar las trampas. Más de la mitad (62.5\%) de los participantes se refirieron a la necesidad de combinar diferentes técnicas y estar al día con soluciones de software de seguridad innovadoras. El navegador Securexam, por ejemplo, impide que los estudiantes abran cualquier otra ventana durante un examen en línea y desactiva las opciones del botón derecho, como copiar, pegar y capturar la pantalla (Anusha et al., 2012; Sarrayrih y llyas, 2013).

La autenticación de los examinados es otro aspecto importante de la seguridad, como señalaron casi dos tercios de los participantes. La mera exigencia de introducir un nombre de usuario y una contraseña no es suficiente para este propósito. Las tecnologías de detección, como las cámaras web, el análisis biométrico de las pulsaciones del teclado y otros sofisticados programas informáticos, están disponibles para apoyar la vigilancia y la autenticación, lo que permite al sistema verificar la identidad de los estudiantes y validar sus logros. Sin embargo, la autentificación de la identidad y el control son más dificil en el caso de los exámenes en línea a distancia, la autenticación de la identidad y el control son más difíciles, ya que, a diferencia de lo que ocurre en un aula con la presencia física de un supervisor, los estudiantes a distancia suelen realizar los exámenes en entornos no controlados, como casas o lugares públicos. Por lo tanto, para demostrar y mantener la integridad, las universidades requieren vigilancia en tiempo real. El software de supervisión remota permite a los estudiantes realizar los exámenes en cualquier lugar que elijan, supervisando la manipulación del ratón y los movimientos de la cabeza y los ojos para detectar los intentos de engaño. 
Aunque Moodle no dispone de funciones totalmente eficaces para tratar las trampas y el plagio. Proporciona una amplia gama de opciones para aleatorizar el orden de las preguntas de opción múltiple y barajar las respuestas, minimizando así el comportamiento poco ético. La tabla 1 muestra que el $75.4 \%$ de los participantes está de acuerdo en que las preguntas aleatorias de un banco hacen que sea menos probable hacer trampas durante los exámenes en línea que en los de papel, lo que coincide con las conclusiones de Bernik y Jereb (2006).

\section{Conclusiones}

Con el uso generalizado y creciente de exámenes en línea en instituciones de educación superior en Perú, este estudio ha examinado los aspectos prácticos de dichos exámenes e identificado varios factores a considerar para apoyar la implementación exitosa de exámenes en línea desde la perspectiva de los estudiantes de la Universidad Alas Peruanas.

Los participantes percibieron que los exámenes en línea tenían ventajas significativas sobre los tradicionales basados en papel, incluida la fiabilidad de la puntuación y la eficiencia a largo plazo en términos de tiempo, esfuerzo y costos. Los hallazgos también indican que los aspectos de equidad, validez y seguridad son los principales desafíos a los que se enfrenta la implementación exitosa de exámenes en línea.

Por lo tanto, la eficacia de los exámenes en línea se puede lograr mediante el diseño de los mismos para que sean válidos, fiables, seguros y flexibles, con el propósito de promover el aprendizaje y asegurar la alineación con los resultados de aprendizaje previstos. La implementación exitosa requiere apoyo institucional, incluida la creación de condiciones adecuadas para la realización de exámenes en línea, facilitando los trámites administrativos, proporcionando el apoyo financiero necesario, mejorar la infraestructura, la creación de la capacidad del personal académico y proporcionarles orientación y apoyo técnico y pedagógico. Este estudio ha demostrado que, en aras del desarrollo sostenible, el enfoque de examen en línea debe integrarse dentro de la planificación estratégica de la Universidad.

\section{Bibliografía}

Anusha, N. S., Soujanya, T. S., \& Vasavi, D. S. (2012). Study on techniques for providing enhanced security during online exams. International Journal of Engineering Inventions, 1(1), 32-37.

Ayo, C. K., Akinyemi, I. O., Adebiyi, A. A., \& Ekong, U. O. (2007). The prospects of e-examination implementation in Nigeria. Turkish Online Journal of Distance Education, 8(4), 125-134.

Baleni, Z. G. (2015). Online formative assessment in higher education: Its pros and cons. Electronic Journal of e-Learning, 13(4), 228-236. 
Bernik, I. , \& Jereb, E. (2006). Students' readiness for electronic examinations'. En Proceedings of the 5th WSEAS International Conference on Education and Educational Technology (págs. 142-145). Tenerife.

Chua, Y. P., \& Don, Z. M. (2013). Effects of computer-based educational achievement test on test performance and test takers' motivation. Computers in Human Behavior, 29(5), 1889-1895.

Dermo, J. (2009). e-Assessment and the student learning experience: A survey of student perceptions of e-assessment. British Journal of Educational Technology, 40(2), 203-214.

Dreher, C., Reiners, T., \& Dreher, H. (2011). Investigating factors affecting the uptake of automated assessment technology. Journal of Information Technology Education: Research, 10(1), 161-181.

Farzin, S. (2017). Attitude of Students Towards E-Examination System: an Application of E-Learning. Science Journal of Education, 4(6), 222.

Gershon, R. C. (2005). Computer adaptive testing. Journal of applied measurement, 6(1), 109-127.

Heinrich, E., Milne, J., \& Moore, M. (2009). An investigation into e-tool use for formative assignment assessment-status and recommendations. Journal of Educational Technology \& Society, 12(4), 176-192.

Hernández Sampieri, R., Fernández Collado, C., \& Baptista Lucio, P. (2010). Metodología de la Investigación (Sexta ed.). Madrid: McGraw Hill.

Hodgson, P., \& Pang, M. Y. (2012). Effective formative e-assessment of student learning: a study on a statistics course. Assessment \& Evaluation in Higher Education, $37(2), 215-225$.

James, R. (2016). Tertiary student attitudes to invigilated, online summative examinations. International Journal of Educational Technology in Higher Education, 13(1), 1-13.

Jamil, M., Tariq, R. H., \& Shami, P. A. (2012). Computer-based vs paper-based examinations: Perceptions of university teachers. Turkish Online Journal of Educational Technology-TOJET, 11(4), 371-381.

Jordan, S. (2013). E-assessment: Past, present and future. New Directions in the Teaching of Physical Sciences, 9(1), 87-106.

Kuikka, M., Kitola, M., \& Laakso, M. J. (2014). Challenges when introducing electronic exam. Research in Learning Technology, 22(1), 1-17.

McGarvey, D. J., \& Haxton, K. J. (2011). Using audio for feedback on assessments: Tutor and student experiences. New Directions in the Teaching of Physical Sciences, 7 , 5-9.

Nicol, D. (2007). E-assessment by design: using multiple-choice tests to good effect. Journal of Further and higher Education, 31(1), 53-64.

Osuji, U. S. (2012). The use of e-assessments in the Nigerian higher education system. Turkish Online Journal of Distance Education, 13(4), 140-152.

Sarrayrih, M. A., \& llyas, M. (2013). Challenges of online exam, performances and problems for online university exam. International Journal of Computer Science Issues (IJCSI), 10(1), 439-443. 
Shraim, K., \& Crompton, H. (2015). Perceptions of Using Smart Mobile Devices in Higher Education Teaching: A Case Study from Palestine. Contemporary Educational Technology, 6(4), 301-318.

Sorensen, E. (2013). Implementation and student perceptions of e-assessment in a Chemical Engineering module. European Journal of Engineering Education, 38(2), 172-185.

Whitelock, D. (2006). Electronic assessment: marking, monitoring and mediating learning. International Journal of Learning Technology, 2(2-3), 264-276. 